\title{
Brain degeneration induced by psychosocial stress
}

\author{
ERNEST GREENE and JENNINGS NEAL NARANJO \\ University of Southern California, Los Angeles, California
}

\begin{abstract}
Psychological and social stress can produce irreversible damage to various organs and physiological control systems. It is understood that the brain plays a role in responding to stressful conditions, but up to now there has been no clear evidence that stress, in the young animal, can produce irreversible damage to the brain. Here we report degeneration of axons of mice as a correlate of stress-induced hypertension, the loss of fibers being most conspicuous in the optic tract and in structures related to the hippocampus. It seems likely that hypertension is causing degeneration of axons by a breakdown of the blood-brain barrier, or by changes in the vasculature that predispose the brain to ischemia.
\end{abstract}

There is ample evidence that psychological and social stress have a strong influence on physiological systems, and that they can cause deterioration in and irreversible damage to organs. In mice, for example, the demands of living in a social colony can cause hypertension, arteriosclerosis, myocardial fibrosis, increases in heart and adrenal weight, and renal damage (Henry \& StephensLarson, 1983). Aggressive encounters among the mice lead to the arousal of autonomic and humoral control systems that allow the animals to cope with the demands of the situation. Although the arousal may be viewed as an adaptive response, the existence of organ pathology indicates some element of adverse consequence. Stress can exacerbate the traumatic effects of hypoxia/ischemia, seizure, exposure to antimetabolites, and aging (Sapolski, 1986a, 1986b), but there has been minimal evidence that the brain of a young animal is directly damaged by stress. Here we report such an effect, finding degeneration of axons as a correlate of hypertension in colony-reared mice.

\section{METHOD}

The subjects (CBA/USC mice) were subjected to social stress or control treatments, using the methods developed by Henry and associates (Henry, Meehan, \& Stephens, 1967). In this model, a mixed-sex colony of animals is housed in a set of chambers that are interconnected by narrow tubes. The males of such a colony compete to establish dominance, which results in hypertension and in pathology of their organs. In the present study, two different colony configurations were used-the Henry-Stephens (H/S) complex population cage, and the Reimer-Petras $(\mathrm{R} / \mathrm{P})$ population cage--to provide the animals with differential stress influences (see Figure 1).

For the H/S colony, the protocols for inducing social stress followed exactly the procedure described by Henry et al. (1967). The H/S colony consisted of 16 males and 16 females. Immediately after being weaned,

This work was supported in part by the Neuropsychology Foundation. Correspondence should be addressed to Ernest Greene, Department of Psychology, Seely G. Mudd Building, University of Southern California, Los Angeles, CA 90089. the mice were raised in isolation for a period of 4 months before being placed in the social stress colony. They were then maintained in the colony for a period of 7 months. Animals in the $\mathrm{H} / \mathrm{S}$ control condition were housed in standard polycarbonate cages (6-8 same-sex siblings per cage) throughout the 11-month period.

For the R/P stress condition, the mice were raised in the standard cages for 13 weeks after weaning (6-8 same-sex siblings per cage); then they were transferred to one of three identical R/P population cages for a period of 4 months. Animals in the R/P control condition were kept in the standard laboratory cages for 7 months.

In order to confirm the presence of hypertension in the males, they were removed from the stress colonies twice a month, and their blood pressure was recorded with a tail plethysmograph.
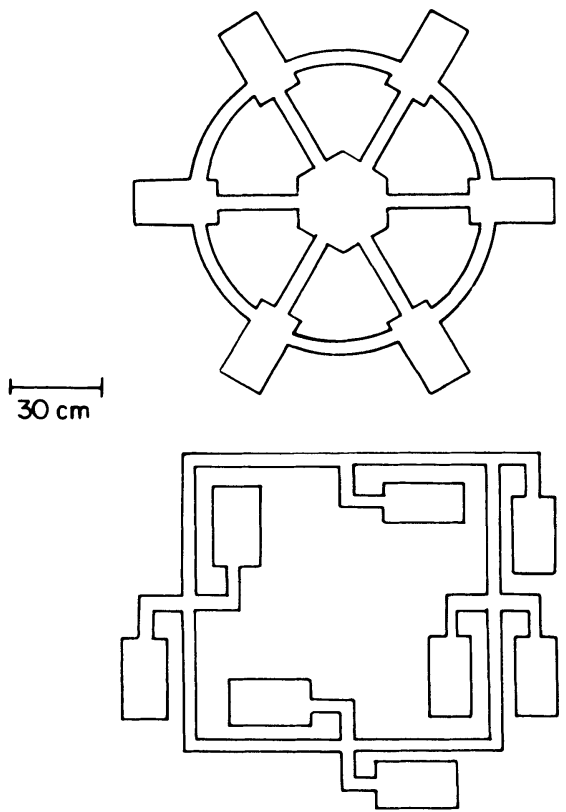

Figure 1. The configuration of the Henry-Stephens population cage is shown at the top, and that of the Reimer-Petras population cage is shown at the bottom. The cages provide differential opportunities for males to establish and defend territories, as well as vary the requirements for social confrontation when they attempt to get food and water. 
Some animals did not survive the trauma of the social confrontations, being found dead in the colony, or with such wounds that humane considerations required that they be removed from the study. The data from these animals were discarded, and so the results reported below are from the animals that were able to cope with the social stress over a period of many months. Most fights occurred early in this period, with 1 male becoming dominant in his encounters and with others either failing to survive or becoming subordinate. Once dominance was determined, there were relatively few overt fights, and the animals seemed reasonably healthy.

At the end of the social stress (or control) exposure period, the animals were killed, and the brains of the males were sectioned and stained, using a reduced silver technique that shows the presence of degenerating axons (Greene \& Naranjo, 1987).

\section{RESULTS}

As is shown in Figure 2, there was a significant increase in the average systolic blood pressure for mice housed in the $\mathrm{H} / \mathrm{S}$ and $\mathrm{R} / \mathrm{P}$ stress colonies. The blood pressure


Figure 2. Changes in mean systolic blood pressure are shown for male mice subjected to social stress or control conditions. The data for the H/S population colony and control subjects are shown at the top; the blood pressure is significantly higher in stressed animals after being housed for 7 months in the social colony. Similar changes are produced after $\mathbf{4}$ months of social stress in the R/P colony (shown at the bottom). of subjects in the $\mathrm{H} / \mathrm{S}$ colony rose from an initial systolic level of $126 \pm 12 \mathrm{mmHg}$ to a mean systolic level of 171 $\pm 19 \mathrm{mmHg}$. Likewise, the blood pressure of the mice housed in the R/P colonies increased to hypertensive levels $(158 \pm 18 \mathrm{mmHg})$ after 4 months of social stress. The blood pressure of the control animals remained in the normal range (120-128 $\mathrm{mmHg}$ ).

Degeneration of fiber tracts was a conspicuous feature of the brains of the socially stressed animals. The appearance of fiber degeneration varies somewhat, depending on the size of the damaged fibers, the time since damage, and other factors. In general, it is recognizable by the presence of black spheroid particles scattered throughout the affected area, as is shown in Figure 3. Reduced silver stain is a reliable method for showing the presence of degenerating axons (Giolli \& Karamanlidis, 1978), with most disputes about stain characteristics relating to the identification of synaptic terminals. We have systemically varied stain procedures, and have not been able to produce the degeneration markers in undamaged tissue (Greene \& Naranjo, 1987).

The brains were examined, with systematic observations of structures listed in Tables 1 and 2. For each animal, the sampled areas were scored by assigning the values of $0,+$, and ++ , representing minimal, typical, and conspicuous levels of degeneration. Although this scoring method is only semiquantitative, it appears to be consistent, as is evidenced by a high between-observer reliability coefficient $(r=.80, F=32.1, p<.001)$.

A sign test of the values in Tables 1 and 2 indicated that the stressed animals differed from the control animals ( $p<.004$ for each colony design). In addition, there was a significant correlation between the level of hypertension and the severity of neural degeneration (for $\mathrm{H} / \mathrm{S}, r=$ $.80, p<.001$; for $\mathrm{R} / \mathrm{P}, r=.85, p<.001)$.

Over time, degenerating fibers first appear as beaded strings, and then the strings become broken and irregular as glial cells remove debris through the process of phagocytosis. In a given stressed brain, one could see the various stages of degeneration debris. A given tract might appear uniform - it might have, for example, a regular, beaded appearance, suggesting that the degenerationinducing trauma had occurred only a few weeks prior to sacrifice. In other tracts, one could see a mixture of several degeneration stages. This suggests that stressinduced damage takes place across time, either as a slow, protracted process, or as an accumulation of multiple traumatic events.

\section{DISCUSSION}

It is possible that the axon degeneration we observed was due to hypertensive encephalopathy, in which bouts of high blood pressure cause a break-down of the blood-brain barrier, edema, and tissue damage (Johansson, 1976; Van Deurs, 1980). The traditional view of hypertensive encephalopathy is that a rapid rise of blood pressure produces vasospasm of the arteries and/or arterioles serving the brain, resulting in edema (Marshall, Jackson, \& Langfitt, 1969) and ischemia in the regions they serve (Gifford \& Westbrook, 1974). Alternatively, there are indications that the rise in blood pressure may cause direct breakdown in the blood-brain barrier (Johansson, Strandgaard, \& Lassen, 

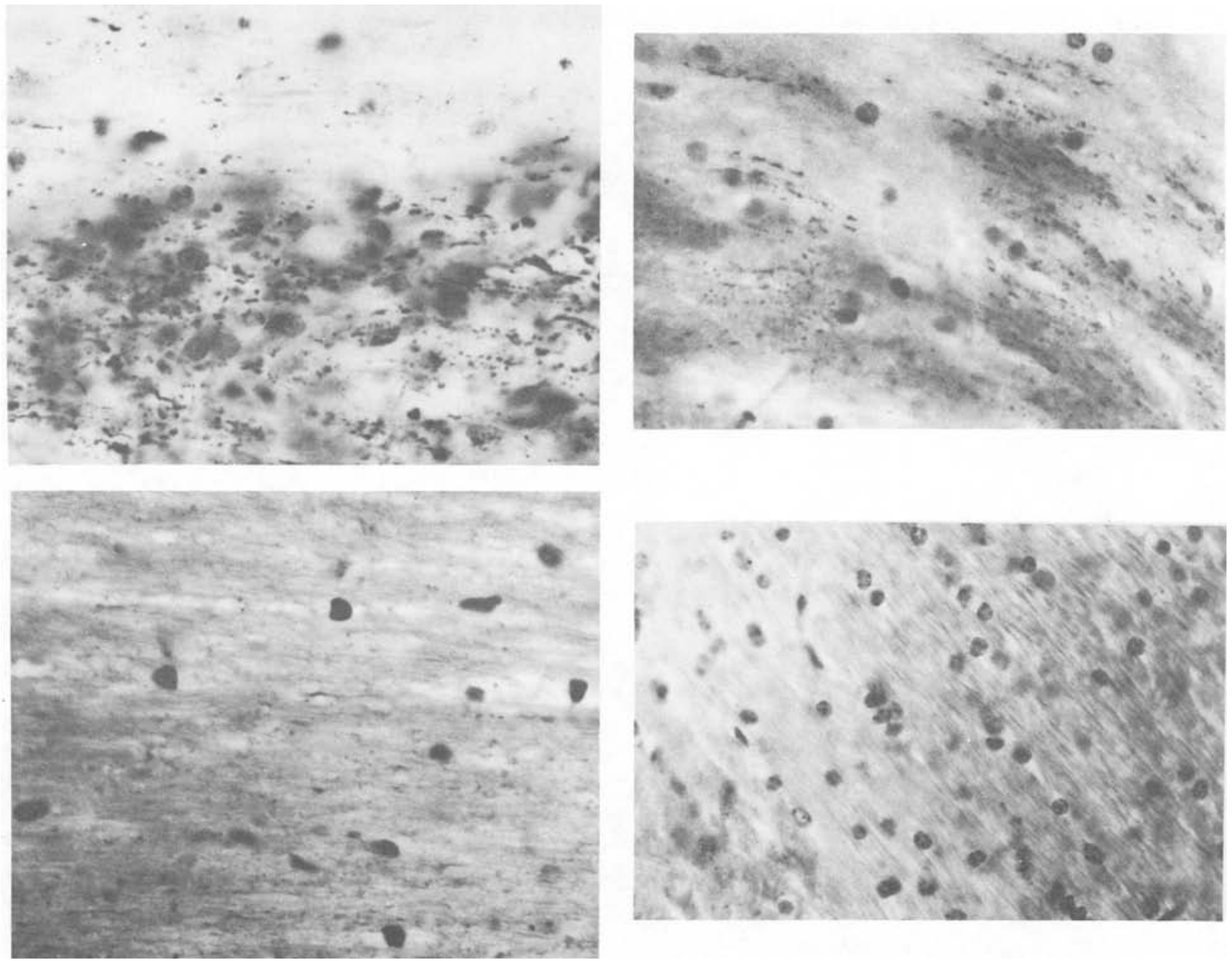

Figure 3. Using reduced silver stain, it is possible to show the presence of degeneration in the brains of the stressed mice: (A) fiber degeneration in the optic tract. (B) beaded strings of degeneration in the fornix. (C) and (D): lack of degeneration in the optic tract and fornix (respectively) of control subjects.

1974; Nag, 1986), allowing ions, serum protein, and humoral agents to penetrate and traumatize the tissue. Temporary breakdown of the blood-brain barrier has been reported with pressure elevations as low as $160 \mathrm{mmHg}$ (Ziylan \& Gokhan, 1985). The stressed mice in the present study had average blood pressures in excess of this value, and with social confrontation one might expect acute pressures of $200 \mathrm{mmHg}$ or more.
Alternatively, chronic hypertension may bring about structural changes in vessels, thus affecting the adequacy of the blood supply. In the brains of spontaneously hypertensive rats, there is a thickening of artery walls and a decrease in the diameter of the lumen (Nordborg, Fredriksson, \& Johansson, 1985). A decrease in the number of arteries per unit area has been reported with several different models of hypertension (Sokolova et al., 1985). These changes may leave the brain vulnerable to drops

Table 1

Evaluation of the Extent of Degeneration in Sampled Brain Areas for Subjects of the Henry-Stephens Stress and Control Conditions

\begin{tabular}{|c|c|c|c|c|c|c|c|c|c|c|c|c|c|c|c|c|}
\hline \multirow[b]{2}{*}{ Brain Area } & \multicolumn{8}{|c|}{ H/S Stressed Subjects } & \multicolumn{8}{|c|}{ H/S Control Subjects } \\
\hline & 5 & 7 & 8 & 10 & 11 & 12 & 14 & 15 & 21 & 22 & 23 & 24 & 25 & 26 & 27 & 28 \\
\hline Optic tract & + & ++ & ++ & ++ & ++ & ++ & $+t$ & ++ & 0 & 0 & 0 & 0 & 0 & 0 & 0 & 0 \\
\hline Corpus callosum & + & + & + & + & + & ++ & + & + & 0 & 0 & 0 & 0 & 0 & 0 & 0 & 0 \\
\hline Fornix & + & + & + & + & + & + & + & + & 0 & 0 & 0 & 0 & 0 & 0 & 0 & 0 \\
\hline Fimbria & + & + & + & ++ & + & + & + & + & 0 & 0 & 0 & 0 & 0 & 0 & 0 & 0 \\
\hline Hippocampus & + & + & + & + & + & + & + & + & 0 & 0 & + & 0 & 0 & 0 . & 0 & 0 \\
\hline Internal capsule & + & + & + & + & + & + & + & + & 0 & 0 & 0 & 0 & 0 & 0 & 0 & 0 \\
\hline Neocortex & + & + & + & + & + & + & + & + & 0 & 0 & + & 0 & 0 & 0 & 0 & 0 \\
\hline Hypothalamus & + & + & + & + & + & + & + & + & 0 & 0 & 0 & 0 & 0 & 0 & 0 & 0 \\
\hline
\end{tabular}

Note-Sign test was used to establish that the stress animals had significantly more brain degeneration than did the control animals. $+=$ fine beaded initial degeneration. $++=$ heavy degeneration. $0=$ no evidence of degeneration. 
Table 2

Evaluation of the Extent of Degeneration of the Sampled Brain Areas for the Reimer-Petras Stress and Control Conditions

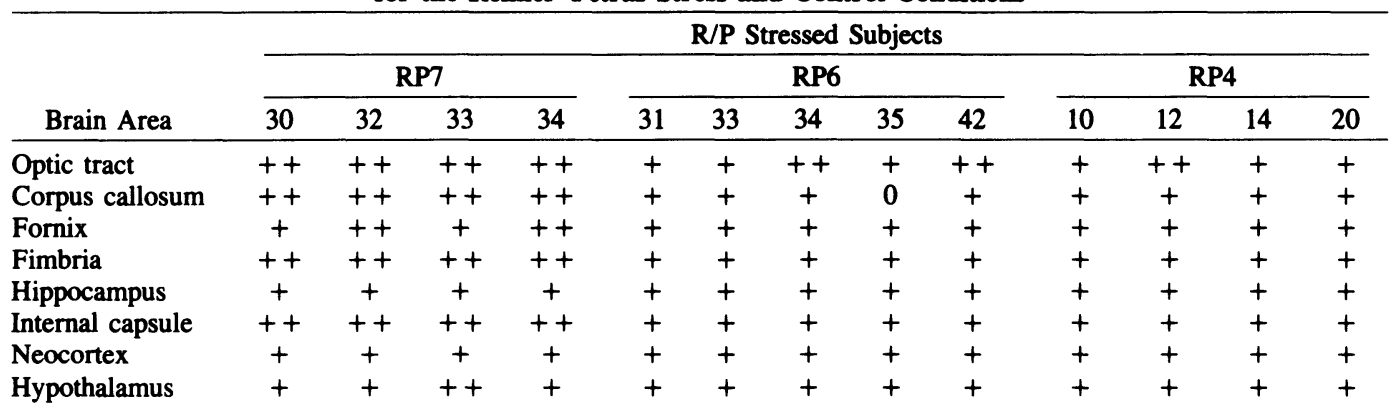

R/P Control Subjects

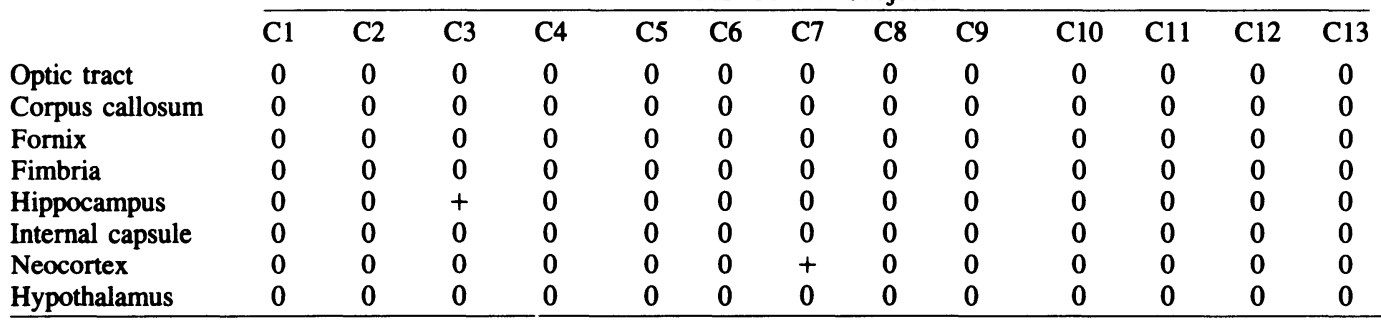

Note-The data for the three R/P colonies are shown separately, but statistical evaluation was done taking all the stressed animals as a single group. Levels of degeneration were significant, relative to those for controls. $\quad+=$ fine beaded initial degeneration. $++=$ heavy degeneration. $0=$ no evidence of degeneration.

in blood pressure (Johansson, 1984). In the present case, the social confrontation might have produced extreme swings in blood pressurewith high levels during a fight, followed by post-traumatic depression. Under these conditions, structural changes in arteries could cause substantial underperfusion of the tissue, hypoxia, and damage to the cells.

The present results indicate a degeneration of axons, and the appearance of the white matter is similar to what would be seen if the axons were directly traumatized. Although we cannot rule out subtle damage to cellular areas, we suspect that the axons are more vulnerable than are the cell bodies per se. A number of experimental and clinical studies of head trauma suggest that the fiber tracts are most susceptible to damage, with axons showing reactive changes from mild concussion that has no observable influence on the ultrastructure of cell bodies or dendrites (Povlishock, 1986). In addition, we have previously reported the degeneration of fiber tracts in normal aged rats (Greene \& Naranjo, 1987), and the pattern of loss is quite similar to the effects of social stress/hypertension reported here. In the context of the present results, it seems likely that age and systemic trauma contribute in an interactive way to the decline of brain function, and that the degeneration of axons may provide an early indicator of this deterioration.

\section{REFERENCES}

Gifford, R. W., \& WESTBRoOK, E. (1974). Hypertensive encephalopathy: Mechanisms, clinical features, and treatment. Progress in Cardiovascular Disease, 17, 115-124.

Giolli, R. A., \& Karamanlidis, A. N. (1978). The study of degenerating fibers using silver-impregnation methods. In $\mathbf{R}$. T. Robertson (Ed.), Neuroanatomical research techniques (pp. 211-240). New York: Academic Press.

GreEne, E., \& NARANJo, J. N. (1987). Degeneration of hippocampal fibers and spatial memory deficit in the aged rat. Neurobiology of $\mathrm{Ag}$ ing, 8, 35-43.

Henry, J. P., Meehan, J. P., \& Stephens, P. M. (1967). The use of psychosocial stimuli to induce prolonged systolic hypertension in mice. Psychosomatic Medicine, 29, 408-432.

Henry, J. P., STEPhens-Larson, P. M. (1983). Specific effects of stress on disease processes. In G. P. Moberg (Ed.), Animal stress (pp. 161-175). Baltimore: American Physiological Society.
JoHANsson, B. B. (1976). Brain barrier pathology in acute arterial hypertension. In G. Levi, L. Battistin, \& A. Lajtha (Eds.), Transport phenomena in the nervous system (pp. 517-527). New York: Plenum.

Johansson, B. B. (1984). Cerebral vascular bed in hypertension and consequences for the brain. Hypertension, 6(Suppl. 3), 81-86.

Johansson, B., StrandgaARd, S., \& LASSEN, N. A. (1974). Encephalopathy-Hypertensive breakthrough of autoregulation of cerebral blood flow with forced vasodilation, flow increase and bloodbrain barrier damage. Circulation Research, 34/35(Suppl. 1), 167-174.

Marshall, W. J. S., Jackson, J. L. F., \& Langfitt, T. W. (1969). Brain swelling caused by trauma and arterial hypertensionHemodynamic aspects. Archives of Neurology, 21, 545.

NAG, S. (1986). Cerebral endothelial plasma membrane alterations in acute hypertension. Acta Neuropathologica, 70, 38-43.

Nordborg, C., Fredriksson, K., \& Johansson, B. (1985). The morphometry of consecutive segments in cerebral arteries of normotensive and spontaneously hypertensive rats. Stroke, 16, 313-320.

PovusHoCK, J. T. (1986). Traumatically induced axonal damage without concomitant change in focally related neuronal somata and dendrites. Acta Neuropathologica, 70, 53-59.

SAPOLSK, R. M. (1986a). Glucocorticoid toxicity in the hippocampusReversal by supplementation with brain fuels. Journal of Neuroscience, 6, 2240 .

SAPOLSK, R. M. (1986b). Glucocorticoid toxicity in the hippocampusTemporal aspects of synergy with kanic acid. Neuroendocrinology, 43, 440-444.

Sokolova, I. A., Manukhina, E. B., Blinkov, S. M., Koshelev, V. B., Pineuis, V. G., \& Rodionov, I. M. (1985). Rarefication of the arterioles and capillary network in the brain of rats with different forms of hypertension. Microvascular Research, 30, 1-9.

VAN DEURS, B. (1980). Structural aspects of brain barriers with special reference to the permeability of the cerebral endothelium and choroidal endothelium. International Review of Cytology, 65, 117-191.

ZIYLAN, Y. Z., \& GoKHAN, N. G. (1985). Water and electrolyte content of rabbit brain after opening the blood-brain barrier by acute hypertension. Experimental Neurology, 87, 198-205.

(Manuscript received October 20, 1989.) 